\title{
DAS BUCH UND SEIN STELLENWERT IM DEUTSCHSPRACHIGEN EXIL 1933-1945
}

Schlüsselworte: deutschsprachiges Exil, Exilliteratur, Bücherverbrennung

Das deutschsprachige literarische Exil während der nationalsozialistischen Diktatur kann zwar keineswegs als homogene Gruppe gedacht werden, doch finden sich in ihm gehäuft bestimmte Problematiken und gemeinsame Interessen, die bei der Literaturproduktion eine essentielle Rolle spielen. Eine davon ist eine verstärkte Konzentration auf das Buch in den Texten, wofür der Beitrag zentrale Beispiele präsentieren will. Daraufhin sollen die Ursachen dieser Betonung dargelegt und diskutiert werden.

\section{Das Buch als zentraler Protagonist in der Exilliteratur}

Auszüge aus der Schachnovelle (1943) Stefan Zweigs sollen als erstes Beispiel dienen, denn sie stellen eine einmalige Hymne auf das Buch dar. Der als ehemaliger Vermögensverwalter des österreichischen Adels und Klerus in Gestapohaft geratene Dr. B. erzählt in einer eingefassten Geschichte von der psychologischen Folter, die er in einem Hotelzimmer zu ertragen hatte: „Und immer um mich nur ein Tisch, der Schrank, das Bett, die Tapete, das Fenster, keine Ablenkung, kein Buch, keine Zeitung, kein fremdes Gesicht, kein Bleistift, um etwas zu notieren, kein Zündholz, um damit zu spielen, nichts, nichts, nichts" (Zweig, 2003, 61). Nicht zufällig wird das Buch zuallererst 
genannt und scheinbar sehnsüchtiger vermisst als der Kontakt mit Personen. Sogar ein Kalenderblatt wird ihm in der Isolationshaft zum Faszinosum: ,und ich vermag Ihnen nicht zu erklären, wie in meinem Hunger nach Gedrucktem, nach Geschriebenem ich diese eine Zahl, diese wenigen Worte ,27. Juli an der Wand anstarrte und anstarrte; ich fraß sie gleichsam in mein Gehirn hinein“ (Zweig, 2003, 65). Ein Buch bedeutet dann die ambivalente Rettung aus der psychologischen Foltersituation: Dr. B. scheint zwar vor einem Verhör zur Kundgabe aller seiner Geheimnisse bereit, doch ein Ausweg zeichnet sich in Form eines Buchs in einem im Wartesaal aufgehängten Mantel ab:

Ich trat näher heran und glaubte an der rechteckigen Form der Ausbuchtung zu erkennen, was diese etwas geschwellte Tasche in sich barg: ein Buch! Mir begannen die Knie zu zittern: ein BUCH! Vier Monate lang hatte ich kein Buch in der Hand gehabt und schon die bloße Vorstellung eines Buches, in dem man aneinandergereihte Worte sehen konnte, Zeilen, Seiten und Blätter, eines Buches, aus dem man andere, neue, fremde, ablenkende Gedanken lesen, verfolgen, sich ins Hirn nehmen könnte, hatte etwas Berauschendes und gleichzeitig Betäubendes. (Zweig, 2003, 67)

Die Vorstellung eines Buchs vereinnahmt Dr. B. zur Gänze. Das imaginäre Buch nimmt Besitz von all seinem Denken, seinen Hoffnungen und sogar von seinem Körper.

Schon der Gedanke, ein Buch durch den Stoff mit den Händen wenigstens antasten zu können, machte mir die Nerven in den Fingern bis zu den Nägeln glühen. [...] Ich tastete den Stoff an und fühlte tatsächlich durch den Stoff etwas Rechteckiges, etwas, das biegsam war und leise knisterte - ein Buch! Ein Buch! Und wie ein Schuß durchzuckte mich der Gedanke: stiehl dir das Buch! Vielleicht gelingt es, und du kannst dir's in der Zelle einstecken und dann lesen, lesen, lesen, endlich wieder einmal lesen! Der Gedanke, kaum in mich gedrungen, wirkte wie ein starkes Gift; mit einemmal begannen mir die Ohren zu brausen und das Herz zu hämmern, meine Hände wurden eiskalt und gehorchten nicht mehr. (Zweig, 2003, 68f.)

Bekannterweise folgt die große Enttäuschung als sich wieder zurück im Haftzimmer herausstellt, dass es sich um ein Schachrepetitorium handelt, das zwar lange intellektuelle Anregung bietet, aber zum Nervenzusammen- 
bruch führt. Das Buch wird dabei zum Subjekt, zu einem zentralen Protagonisten der Novelle, ein Akteur, der im Mittelpunkt der Sehnsucht des Gefangenen steht.

Die Reihe der Anführungen von Büchern und Literatur und ihre immense Bedeutung in den Werken des Exils kann lange fortgeführt werden. So liest in Anna Seghers Roman Transit (1943) der Protagonist, ein Monteur und ehemaliger KZ-Häftling ohne jeglichen Bezug zur Kunst, aus Langeweile die zufällig von einem Selbstmörder geerbten Manuskripte.

Ich vergaß meine tödliche Langeweile. Und hätte ich tödliche Wunden gehabt, ich hätte auch sie im Lesen vergessen. Und wie ich Zeile um Zeile las, da spürte ich auch, dass das meine Sprache war, meine Muttersprache, und sie ging mir ein wie die Milch dem Säugling. Sie knarrte und knirschte nicht wie die Sprache, die aus den Kehlen der Nazis kam, in mörderischen Befehlen, in widerwärtigen Gehorsamsbeteuerungen, in ekligen Prahlereien, sie war ernst und still. (Seghers, 2001, 25)

Das Buch vermag auch hier einen Lesenden in seinen Bann zu ziehen. Als das gelesene Manuskript in Transit unfertig abbricht, fühlt sich der Leser schrecklich allein gelassen und kann den Selbstmord des Schriftstellers nicht verstehen. Auch wenn er als Knabe nie gern gelesen hat, wäre ihm nun der unvollendete, aber gefährliche Text wichtiger als ein Menschenleben: „Auf den Pont d'Alma gehn und ihn in die Seine werfen? Da hätte ich lieber ein Kind ertränkt!“ (Seghers, 2001, 28). Auch dieser Text scheint personalisiert, ein Helfer in der Not und Langeweile, ein Identitätstifter, der ebenso eine Gefahr darstellt.

Selbst Pessimisten wie Joseph Roth können sich der Faszination des Buches - im Fall des Journalisten Roth auch der Zeitung - in keinem Werk entziehen, wobei immer wieder unterstrichen wird, dass die Protagonisten der Romane und Erzählungen nicht lesen. In seiner berühmten Legende vom heiligen Trinker (1939), taucht naturgemäß kein intellektueller Diskurs zum Buch im gezeichneten Obdachlosen- und Trinkermilieu auf. So dienen Andreas Kartak Zeitungen zu einem spezifischen Zweck: „Ja, er klaubte sich sogar noch eine Zeitung aus einem Papierkorb auf. Aber nicht um in ihr zu lesen, sondern, um sich mit ihr zuzudecken. Denn Zeitungen halten 
warm, das wissen alle Obdachlosen“ (Roth, 1999, 11). Zu Geld gekommen bleibt er am Kiosk stehen und kauft sich eine Zeitung, aber nur um zu erfahren, welches Datum und welcher Wochentag sei.

Das Buch bekommt in diesem Werk ebenso eine skurrile Funktion. Im Hotel lernt Andreas eine Gabby kennen, sie fassen ein Rendezvous ins Auge, doch Andreas will nicht so lange warten und geht in ihr Zimmer. Gabby ist zwar nicht überrascht, als er in ihr Zimmer kommt, doch sie heuchelt Beschäftigtheit und Überraschung, indem sie ein längst gelesenes Buch als Barriere und Schutz zur Hand nimmt, worauf Andreas „aufrichtig“ meint: "Ich interessiere mich nicht für Bücher" (Roth, 1999, 43). Sogar die Werke des Skeptikers Roth handeln immer wieder explizit von Büchern, wenn auch zumeist vom Nichtlesen.

\section{Das Buch als psychologische Stütze im Exil}

Die Ursachen für diese Schwerpunktlegung der Exilliteratur auf das Buch und den geschriebenen Text ist mannigfaltig; ein Grund ist psychologischer Natur. Den geflohenen Schriftstellern wurde ihr ganzes Publikum, der lebensnotwendige Literaturbetrieb und der kulturelle wie sprachliche Nährboden ihrer Arbeit entzogen. Nicht einmal Briefe konnten bald mit Deutschland ausgetauscht werden, um die Daheimgebliebenen nicht zu gefährden. Das mitgebrachte, neu zugelegte oder sogar im Exil verlegte deutsche Buch konnte diese Isolation zeitweilig durchbrechen.

Doch nicht nur Bücher bekamen einen neuen Stellenwert. Die deutsche Sprache wurde in einem neuen Licht gesehen, der Nationalsozialismus an der Regierung verursachte einen Reflektionsprozess zur eigenen Kultur und Sprache, einer der häufigsten Themen in der Lyrik - so in Berthold Viertels Die deutsche Sprache (1941):

\footnotetext{
„Dass ich bei Tag und Nacht

In dieser Sprache schreibe,

Ihr treuer als der Freundschaft und dem Weibe,

Es wird mir viel verdacht." (Kramer, 1994, 177)
} 
Die Schriftsteller blieben zumeist stark an die deutsche Sprache gebunden und wurden nur wenig durch die Literatur des Gastlandes beeinflusst. Günther Anders erläutert, dass viele sogar zu schreiben begannen, „weil die Sprache das einzige Gerät war, mit dessen Hilfe sie [=die Exilanten] sich, wenn auch nicht vor dem physischen Untergang, so doch vor dem letzten Herunterkommen bewahren konnten; und weil sie das einzige unraubbare Gut war, das einzige Stück Zuhause, das sie, wenn sie es verteidigten, selbst im Zustande restloser Entwürdigung noch beherrschten, das einzige, das (wenn auch nur ihnen selbst) bezeugte, wo sie hingehörten“" (Anders, 1962, $621)$.

Für ihre eigene Identität und Selbstachtung sowie manchmal im Sinne ihrer politischen Interessen trennten Exilanten die deutsche Sprache als solche klar von der nationalsozialistischen Rhetorik, Propaganda und Ideologie. Die im bereits erwähnten Zitat aus Anna Seghers Transit formulierte Gegenüberstellung der für die Exilanten identitätsstiftenden Kultur und Sprache, die oft nur noch in Büchern zu finden war, mit der Unkultur der Nazis bleibt ein immer wiederkehrendes Thema im Exil. Sie stellt eine zentrale Basis der Identität der Geflohenen dar.

In Transit wird auch die therapeutische Funktion der Literatur im Exil explizit angesprochen. Die Erzählung in der Muttersprache bedeutet für den Leser Geborgenheit. Doch auch für den Literaten hat das Schreiben selbsttherapeutische Funktionen, der Selbstreflektion und Bewusstwerdung. Klar bezieht der Erzähler dazu Stellung: „Denn abgeschlossen ist, was erzählt wird“ (Seghers, 2001, 215). Diese therapeutischen und selbsttherapeutischen Funktionen waren nach dem psychologischen Schock des Ausgestoßenwerdens und des Unerwünschtseins sowie dem Erleben einer permanenten Entfremdung essentiell.

\section{Reflektionen zu den sozialen und politischen Aufgaben und Möglichkeiten des Buches}

Die Konzentration der Exilliteratur auf das Buch kann darüber hinaus nicht ohne die vertiefte Reflektion über die Aufgaben der Literatur geda- 
cht werden, welche im Exil ausgiebig diskutiert wurden. Als Kommunistin ruft Seghers mit Transit dazu auf, dass Schreiben alleine nicht genüge, um den Faschismus zu bekämpfen. Dieser Roman ist dabei kennzeichnend für die intensiven, insbesondere um den historischen Roman entbrannten Debatten um die Aufgaben der Literatur, die in den Exilzeitschriften, bei Kongressen und in Briefen geführt wurde (Wegner, 1967, 140ff.), wobei einerseits eine moralische, politische und künstlerische Verpflichtung eingefordert wurde, der andererseits (z. T. resignierende) Plädoyers für eine zweckfreie Kunst gegenüberstanden. Bekannt wurde auch die von über 20 Schriftstellern - darunter Georg Lukács, Hanns Eisler, Klaus Mann, Ernst Bloch und Anna Seghers - geführte Expressionismusdebatte, die von marxistischer Seite vor allem in der Moskauer Zeitschrift Das Wort geführt wurde und Fragen zum Verhältnis zwischen Expressionismus und Faschismus und Möglichkeiten realistisch-materialistischer Alternativen nachging. Wie in wohl keiner anderen Epoche wurden die Funktionen der Literatur und die eigene Position des Schriftstellers mit ernstem Enthusiasmus reflektiert und diskutiert.

Weite Teile der - auch vorher unpolitischen - Schriftsteller fühlten sich dazu berufen, gegen das Hitlerregime zu kämpfen, indem sie die Welt über die wahre Natur des Nationalsozialismus aufklärten und die deutsche Kultur in ihrem Sinne bewahrten. Sogar in naiven Romanperspektiven, wie in Irmgard Keuns Nach Mitternacht (1937), taucht im Umfeld der wenig intellektuellen Erzählerin im nationalsozialistischen Deutschland diese Diskussion mit dem Journalisten Heini auf, der den Schriftsteller Algin für seine Anbiederung an das Hitlerregime auf ironische Weise kritisiert:

Einen historischen Roman willst du jetzt schreiben? Als Eunuch wirst du diesen Roman schreiben. Ein Schriftsteller hat sich weder vor den eigenen Sätzen noch vor Gott und der Welt zu fürchten, wenn er schreibt. Ein Schriftsteller, der Angst hat, ist kein Schriftsteller. Aber abgesehen davon: Du bist überflüssig. Durch die Diktatur ist Deutschland ein vollkommenes Land geworden. Ein vollkommenes Land braucht keine Schriftsteller. Im Paradies gibt es keine Literatur. Ohne Unvollkommenheiten gibt es keine Schriftsteller und keine Dichter. Der reinste Lyriker bedarf der Sehnsucht nach Vollkommenheit. Wo Vollkommenheit ist, hört die Dichtung auf. (Keun, 2003, 86) 
Heini betont die Pflicht der Literatur zur Kritik an den Umständen und damit zum Beitrag zu deren Verbesserung. Sonst ist Literatur für ihn sinnlos, er pflichtet so den Suizidgedanken Algins bei: „Bring dich um oder lern Harfe spielen und mach Sphärenmusik“ (Keun, 2003, 87).

Die Reflektion zu Aufgaben des Literaten und der Literatur taucht in verschiedensten Werken und Literaturgattungen auf, wofür auch Bertolt Brechts Gedicht Über die Bezeichnung Emigranten (1937) ein eindrucksvolles Beispiel bietet.
Unruhig sitzen wir so, möglichst nahe den Grenzen
Wartend des Tags der Rückkehr, jede kleinste Veränderung
Jenseits der Grenze beobachtend, jeden Ankömmling
Eifrig befragend, nichts vergessend und nichts aufgebend
Und auch verzeihend nichts, was geschah, nichts verzeihend.
Ach, die Stille der Stunde täuscht uns nicht! Wir hören die Schreie
Aus ihren Lagern bis hierher. Sind wir doch selber
Fast wie Gerüchte von Untaten, die da entkamen
Über die Grenzen. Jeder von uns
Der mit zerrissenen Schuhn durch die Menge geht
Zeugt von der Schande, die jetzt unser Land befleckt.
Aber keiner von uns
Wird hier bleiben. Das letzte Wort
Ist noch nicht gesprochen. (Brecht, 1990, 718)

Dem Schriftsteller bleibt im Exil die bedeutende Rolle des informierten Beobachters, intellektuellen Richters, des Chronisten und Aufklärers, die nur beschränkte Zeit von der Überzeugung des nahenden Umschwungs in Deutschland getragen ist. Alleine seine Anwesenheit im Ausland und seine Tätigkeit wird als Anklage gegen das Hitlerregime definiert.

Das Buch konnte dem Chronisten dabei ein Überdauern gewährleisten, denn man schrieb nicht unbedingt für ein zeitgenössisches Publikum. Diese Notwendigkeit gelangte mitunter frustrierend ins Bewusstsein. In Klaus Manns Roman Vulkan (1939), einer netzwerkartige Übersicht und Analyse über verschiedene Exilantenschichten in unterschiedlichsten Ländern, will der Schriftsteller Martin Korella aus denselben Motiven wie Brechts lyri- 
sches Wir im genannten Gedicht eine große Chronik verfassen und scheitert weniger aufgrund seiner Drogensucht als aufgrund seiner Zweifel daran:

Für wen schreibe ich? - Immer haben Dichter sorgenvoll darüber nachgedacht. Und wenn sie es gar nicht wussten, dann haben sie wohl - hochmütig und resigniert, stolz und verzweifelt - behauptet: Für die Kommenden! Nicht euch, den Zeitgenossen, gehört unser Wort; es gehört der Zukunft, den noch ungeborenen Geschlechtern. Ach, was weiß man von den Kommenden? Welches werden ihre Spiele, was ihre Sorgen sein? Wie fremd sind sie uns! Wir wissen nicht, was sie lieben oder was sie hassen werden. Trotzdem sind sie es, an die wir uns wenden müssen. (Mann, 1995, 194)

Da der zeitgenössische Leser zumeist fehlte, fand die Mehrheit der Exilschriftsteller kaum adäquate Publikationsmöglichkeiten. Bertolt Brecht beschreibt diesbezüglich in Flüchtlingsgespräche (1940) zwei Exilanten, die sich regelmäßig in Helsinkis Bahnhofsrestaurant treffen. Der Intellektuelle Ziffel schreibt seine Memoiren. Da er kein Publikum und keine Leser dafür hat, „lauert“ er auf seinen einzigen Kollegen Kalle, um ein neues Kapitel vorzulesen: „Als er eine Woche lang nicht kam, dachte Ziffel schon, er habe das Kapitel umsonst geschrieben, und stoppte alle weitere Arbeit" (Brecht, 1990, 30).

In Anbetracht einer derart entmutigenden Rezeptionssituation erscheint das Abfassen einer bloß resumierenden Chronik sinnlos, wenn damit nicht zugleich auch zur Tat aufgerufen würde. In Manns Vulkan soll diese Chronikidee, den „Roman der Heimatlosen“, der junge, dem Katholizismus zugeneigte Kikjou vollenden. Ein „Engel der Vertriebenen“ spricht ihm zu: „Seid verwegen! Das Leben, das ihr aufs Spiel setzen könnt, ist keine so große Sache. Mit einem Schwert wurdet ihr vertrieben aus dem Paradies; mit einem Schwerte sollt ihr es zurückerobern. Ihr müsst euch die Heimkehr erkämpfen, ihr Heimatlosen!“ (Mann, 1995, 549). Klaus Mann lässt so keine Zweifel an seiner Einstellung zur politischen Aufgabe des Buches. Eine weitere Figur, die erfolgreiche Schauspielerin Marion von Kammer, gezeichnet nach Manns Schwester Erika, engagiert sich in ihrem Kabarett- und Rezitierprogramm gegen das Hitlerregime und für die deutsche Kultur. Mit ihrem zukünftigen Mann, dem Literaturwissenschafter Prof. Benjamin Abel entbrennt vorerst die typische Exilantendebatte, da er die 
geistigen Werte in der Literatur liebt und sie deswegen nicht benutzen will. Doch er läßt sich von Marions Argumenten und Wesen umstimmen.

Exilliteraten konnten sogar wirklich tatkräftig mit ihrem Wort den alliierten Kampf gegen Hitler unterstützen, so schrieb und sprach Thomas Mann von 1940 bis 1945 Radiosendungen für das Publikum des BCC in Deutschland. Carl Zuckmayer verfasste Berichte für den amerikanischen Geheimdienst zur Vorbereitung der Besatzung. Klaus Mann fertigte als US-Soldat Flugblätter an sowie Propaganda und Texte für Radio und Grabenlautsprecher.

\section{Bücher im nationalsozialistischen Deutschland}

Eine historische Begebenheit, welche die Aufmerksamkeit in einzigartiger Form auf das Buch lenkte, muss bei den Ursachen zum so starken Interesse der Exilliteratur am Buch noch Erwähnung finden: die Bücherverbrennungen am 10. Mai 1933. Obwohl schon in der Weimarer Republik Bücherverbote und Haftstrafen gegen einzelne Autoren durchgeführt wurden, schon 1932 Notverordnungen die Pressefreiheit empfindlich einschränkten und die nationalsozialistische Regierung 1933 zügig Institutionen wie die Abteilung für Dichtkunst der Preußischen Akademie der Künste, ${ }^{1}$ den Schutzverband deutscher Schriftsteller sowie das PEN-Zentrum brutal auf ihre Linie gebracht hatte, galten und gelten die Bücherverbrennungen als sichtbarer und klarer Höhepunkt der Kampagne zur Gleichschaltung der Kultur.

Die Deutsche Studentenschaft und das Reichspropagandaministerium unter Joseph Goebbels organisierte, von mehreren nationalsozialistischen und völkisch-national-konservativen Einrichtungen und Personen unterstützt, eine Bücherhinrichtung in fast allen deutschen Universitätsstädten. ${ }^{2}$

1 Heinrich Mann musste als deren Präsident zurücktreten. Bald wurden andere Mitglieder sogar trotz Loyalitätserklärungen - wie z. B. von Ludwig Fulda, Franz Werfel, Bernhard Kellermann, Georg Kaiser, Alfred Mombert, Fritz von Unruh - entlassen (Treß, 2003, 47).

2 Und zwar: Berlin, Bonn, Braunschweig, Breslau, Darmstadt, Dresden, Erlangen, Franfurt am Main, Gießen, Göttingen, Greifswald, Halle-Wittenberg, Hamburg, Hannover, Hanno- 
„Festredner“ wie Goebbels in Berlin, aber auch Hochschulgermanisten (Verweyen, 2000, 177ff.), wurden im Rundfunk übertragen. Werke marxistischer, jüdischer, liberaler und pazifistischer Literater $u$. a. wurden von Studierenden mit Feuersprüchen „den Flammen übergeben“.

Die Nazis inszenierten die Bücherverbrennungen als Teil einer Tradition mit Bezug auf das Wartburgfest und auf Luthers Verbrennung der päpstlichen Bannandrohungsbulle. Dass es sich aber um eine diese imaginäre historische Reihe klar übersteigende Singularität handelt (Verweyen, 2000, 207f. oder Treß, 2003, 17), zeigt sich darin, dass es sich um einen Teil einer zentral gesteuerten, vierwöchigen, akribisch geplanten Kampagne, der sogenannten „Aktion wider den undeutschen Geist“ handelte. Die Größenordnung - 10.000 Zentner Bücher alleine in Berlin - war einmalig; die Spanne von Philosophie zur Rechtslehre, Literatur zur Pädagogik und Theologie kennt keinen Vergleich; das Ausmaß und die Intensität der Ausgrenzung, die Organisiertheit und Öffentlichkeitswirksamkeit sowie offizielle und breitenwirksame Legitimierung erreichten einzigartige Ausmaße. Außerdem zielte der Grad des Vernichtungswillens klar auf Personen hin. Der berühmte Germanist Albrecht Schöne, damals Präsident der Internationalen Vereinigung für Germanische Sprach- und Literaturwissenschaft, bemerkte bei einer Erinnerungsrede 1983: „Keiner von uns Nachlebenden kann die hier angesagte Bücherverbrennung anders mehr denken als in einer Reihe von Feuerstätten. Nicht ohne den Reichstagsbrand, der ihre vorausging. Nicht ohne den Brand der Synagogen, der ihr nachfolgte. Nicht ohne den Brand des Krieges dann. Nicht ohne die Verbrennungsöfen der Konzentrationslager mehr" (Schöne, 1983, 23).

In der Mehrzahl handelte es sich um Bücher von Verfassern, die noch bis kurz zuvor in Deutschland gewirkt hatten und ein großes Publikum erreichen konnten (Treß, 2003, 29).

Nun waren die Autoren stigmatisiert und ihre berufliche Existenz oft vernichtet.

versch-Münden, Heidelberg, Kaiserlauten, Karlsruhe, Kiel, Köln, Königsberg, Mannheim, Marburg, München, Münster, Nürnberg, Rostock, Salzburg (1938), Worms und Würzburg (Sauder, 1983, 89f.). 
Dieser „öffentliche Hinrichtung des freien Geistes“ (Treß, 2003, 210) folgten weitere Schwarze Listen gegen „schädliches und unerwünschtes Schrifttum“ für Büchereien und Buchhandel. Eine lückenlose Überwachung der Literatur in Deutschland sollte folgen. Das Ministerium für Volksaufklärung und Propaganda unter Joseph Goebbels sollte dafür große Summen zur Verfügung stellen und 2500 Verlagsanstalten, 2300 Buchläden, 3000 Autoren, 50 nationale Preise, 2000 jährliche Neuerscheinungen und 2500 Bibliotheken, darüber hinaus Zeitungen, Zeitschriften und Bühnen, vor allem durch die Reichsschrifttumskammer überwachen (Schwarz, 1983, 309). Fakt ist, dass aus dieser Maschinerie nur ein vernachlässigbarer Teil an irgendwie ernstzunehmender Literatur entstanden ist. Im Gegensatz dazu ist im Exil eine große Zahl bleibender Werke von hoher Qualität geschaffen worden.

\section{Konsequenzen der Bücherverbrennungen im Exil}

International wurden die Bücherhinrichtungen als Skandal gesehen, der das Wesen der Hitler-Herrschaft, den Rückfall in die Barbarei (Z. B. Mayer, 1983, 305) versinnbildlichte.

Für viele bedeutete der 10. Mai das letzte notwendige Signal, um Deutschland zu verlassen. Die systematische Vertreibung wurde mit weiteren Ausbürgerungslisten von Literaten besiegelt, die so in die Staatenlosigkeit gezwungen wurden.

Schock und fassungsloses Staunen prägten dabei die ersten Reaktionen zu den Bücherverbrennungen. Rund fünfzig Jahre später hatten die Augenzeugen wie der Verleger Gottfried Bermann-Fischer Probleme, das Gesehene in Worte zu fassen: „Es war so außerhalb der Vorstellung, daß man in Deutschland, im Land, das den Buchdruck erfand, im Land der Dichter und Denker, Bücher öffentlich und mit Schmähungen den Flammen übergab, und dass dies öffentlich verkündet und propagiert wurde" (Zit. nach Lischeid, 2001, 537).

Wie er erschienen einige betroffene Schriftsteller und Verleger bei den Bücherverbrennungen, so wohnten auch Golo Mann und Erich Kästner dem 
Ereignis bei. Stefan Zweig behielt sogar ein geschenktes Exemplar eines gepfählten, mit einem Nagel durchbohrten Buches. Viele konzentrierten sich auf solche, die Verbrennungen „überlebenden“ Bücher. Thomas und Heinrich Mann berichten von der angeblich großen Zahl der gestohlenen und somit vor dem Flammentod geretteten Büchern (Lischeid, 2001, 193f.). Alfred Kantorowicz erzählte 1935 vom blühenden Schleichhandel mit Büchern, die auf dem nationalsozialistischen Index standen (Haarmann, 1983, 404).

Ein beredtes literarisches Zeugnis, inwieweit Bücher in der Folge im Exil personalisiert und ins Zentrum der Aufmerksamkeit gerückt wurden, dabei als Brückenschlag zur eigenen Vergangenheit und zur deutschen Kultur fungierten, ist Theodor Kramers Stehn meine Bücher... (1943). Selbst die Erinnerung an eine geliebte Person im Heimatland wird über das Gedenken an Bücher gelenkt, die offenbar verboten sind, da der Umschlag weggenommen worden ist, und sogar in der Gefahr schweben, von der Besitzerin oder dem Besitzer verbrannt zu werden. Sie stellen das wichtige Bindeglied zwischen dem Exilanten und der in Deutschland Zurückgebliebenen dar. Das Gedicht kommt immer wieder auf die eingangs erwähnten Bücher zurück:

Stehn meine Bücher, die ich vorm Verreisen dir schenkte, noch auf deinem Bücherbord, das roch nach Leder, Lack und schwarzem Eisen?

(Wir nahmen vielen noch den Umschlag fort.) Kommst du dazu, in ihnen noch zu lesen [...]? $[\ldots]$ hast du nicht manche doch verbrennen müssen, und leihst du heimlich noch die andern aus? [...] stehn meine Bücher, die ich vorm Verreisen dir schenkte, noch auf deinem Bücherbord? (Kramer, 1984, 326)

Wofür das Buch noch stehen kann und was es bedeutet, es zu verbrennen, das sei exemplarisch an der Exilerzählung Tichons Buch (1933) von Hans Scheer gezeigt, in der ein „typischer SA-Mann“ ein eigenes Buch zur Verbrennung abgibt, doch innere Reue empfindet und zum Tatort zurückkehrt: „Tichon hebt den Kopf. Er denkt immer noch an das eine Buch. Er will nicht daran denken. Aber er kam doch zurück und steht nun und steht [...] Dort liegt das Buch, schwarz, kleine zerbrechliche Flocken, die der Wind forttragen wird. Das Buch. Sein Buch, das er gelesen hat 
und liebt, fast so liebt, wie Maria aus der Spinnerei“" (Scheer, 1933, 73). Aufgrund des zerstörten, personifizierten Buches erlebt er einen inneren Wandel, wird sich der Mangelhaftigkeit seines Lebens in der NS-Bewegung bewusst. Er merkt, dass in dem verbrannten Buch mehr gesteckt hat als in den NS-Büchern; das Buch war er, seine wahre Identität:

Im verbrannten Buch stand er. Dicke Tränen kann er nicht vergessen, die er weinte, da er sich las. Er ist nicht weich, aber er spürte damals, daß er etwas erlebte, das viel stärker war als der Vorbeimarsch am Führer. Er war Mensch im Buch. In Goebbels ,Michael' fand er keinen Menschen. Die waren tot und wächsern, schienen ständig den Klumpfuß des Doktors nachzuschleppen. Michael lebt, von ihm aber ist ein Stück verbrannt. Er wird nicht mehr lieben können. Der Wind treibt schwarze Flocken. Das herrliche Wort ist blind; wird morgen Pulver in der Karre des Straßenfegers. Das Feuer und die Menschen: grinsende Menschen aus dem Vorort, die nicht verstanden, was man ihm tat. (Scheer, 1933, 73)

Dem Buch und seiner Verbrennung werden hier die Macht einer Bekehrung zugeschrieben. Diese Erzählung reflektiert das Selbstbewusstsein der Exilgemeinde, welche die Bücherverbrennungen natürlich aufs Schärfste verurteilt. Ernst Tollers Offener Brief an Herrn Goebbels (1933) und seine Reden auf dem Internationalen PEN-Club-Kongress in Dubrovnik fanden als pathetische Replik weltweites Medienecho. Er erhob dabei seine Stimme gegen die Untaten der Nazis, benannte das Leid der Opfer und appellierte zum Widerstand und Glauben an die eigene Stärke. Heinrich Mann äußerte sich 1936: „Der Bücherverbrennung soll man gedenken - um der Ohnmacht willen, die sich erdreistete, Scheiterhaufen zu errichten für Geisteswerke: als ob Geisteswerke nicht feuerfest wären" (Zit. nach Treß, 2003, 209). Ähnlich formulierte Oskar Maria Graf schon am 12. Mai 1933 den Abschluss seines Protest Verbrennt mich!, in dem er in der Wiener Arbeiterzeitung darum bat, dass seine Werke „nicht in die blutigen Hände und die verdorbenen Hirne der neuen Machthaber gelangen“ und verbrannt werden: „Verbrennt die Werke des deutschen Geistes! Er selber wird unauslöschlich sein, wie eure Schmach!“ (Zit. nach Treß, 2003, 209). Anna Seghers zeigte sich anlässlich des zehnten Jahrestages der Bücherverbrennung ebenso optimistisch: „Daß wir den Jahrestag der Bücherverbrennungen fe- 
iern, das allein zeigt, dass das verbotene Buch in dem Scheiterhaufen des 10. Mai statt zu Asche zu werden, geglüht und gehärtet wurde zu einer handfesten Waffe gegen Hitler“ (Zit. nach Treß, 2003, 209). Das - insbesondere marxistische - Konzept vom Buch als Waffe lebte einmal mehr auf.

Das Buch bekam also gerade durch die Verbrennungen im Bewusstsein des Exils eine erhöhte Stellung. Schon am ersten Jahrestag der Bücherverbrennungen gründete Alfred Kantorowicz in Paris die Deutsche Freiheitsbibliothek, die bald 20.000 Bände sammeln sollte (Lischeid, 2001, 37). Mit wahren Massenveranstaltungen begangen den zehnten Jahrestag die Vereinigten Staaten, die ein gutes Jahr zuvor in den Krieg eingetreten waren. Schon im Dezember 1942 wurde eine Ausstellung der verbrannten und verbotenen Bücher in der Public Library New Yorks eröffnet. Am 10. Mai wurde auf aktionistische Weise eine Bücherverbrennung simuliert, bei der Käufer von Kriegsanleihen das Recht hatten, die künstlichen Flammen zu ersticken. Bedeutende Persönlichkeiten aus Politik, Kunst und Wissenschaft sprachen; vor Hunderten amerikanischen Bibliotheken wehte die Flagge auf Halbmast und im ganzen Land präsentierten Bibliotheken in Ausstellungen die in Deutschland verbotenen Bücher.

Zweifellos spielten die Bücherhinrichtungen 1933 eine bedeutende Rolle in der so hohen Wertschätzung des Buches durch die deutschsprachigen Exilanten. Durch die hassvollen Verbrennungen bezeugten die Nationalsozialisten ungewollt die große Macht der Literatur. Rafetseder $(1988,59 f$. unterstreicht gar, dass übergroße Wertschätzung und übergroßer Hass gegen das Buch verwandt sind und in den Zerstörungstaten wohl die Furcht vor einer Überlegenheit des Buches und deren Folgen mitschwingt.

\section{Fazit}

Das Buch erlangte in der Exilliteratur eine hohe Bedeutung sowohl als Sujet als auch hinsichtlich seiner simplen Materialität, was seiner zentralen Stellung im privaten und öffentlichen Leben der Exilanten entsprach. Als psychologische Stütze ermöglichte es ihnen einen weiteren Kontakt mit ihrer vertrauten Sprache und Kultur. Als politisches Medium konnten es 
die Exilanten im Kampf gegen den Faschismus nutzen. Außerdem wurde das Buch zum antifaschistischen Symbol, als die Nationalsozialisten öffentlichkeitswirksam Bücher verbrannten.

Die Anstrengungen der Exilanten, die geistige und literarische Tradition Deutschlands gegen die nazistische Barbarei zu schützen, brachte eine große Zahl von bleibenden Werken hervor, die es in den Kanon und ins Zentrum der Literaturgeschichte schafften, während die Literatur unter den Nationalsozialisten zu Recht als vernachlässigbar gilt.

\section{LITERATUR}

\section{Primärliteratur}

Brecht, B., Die Gedichte von Bertolt Brecht in einem Band, Frankfurt a. M., 1990.

Brecht, B., Flüchtlingsgespräche, Frankfurt a. M., 1990.

Keun, I., „Nach Mitternacht“ mit Materialien, ausgewählt und eingeleitet von Dietrich Steinbach. Stuttgart, et al., 2003.

Kramer, T., Gesammelte Gedichte 1, hg. von Erwin Chvojka, Wien, 1984.

Mann, K., Mephisto, Reinbek bei Hamburg, 1993.

Mann, K., Der Vulkan. Roman unter Emigranten, Reinbek bei Hamburg, 1995.

Roth, J., Die Legende vom heiligen Trinker, Köln, 1999.

Seghers, A., Transit, Berlin, 2001.

Scheer, H., Tichons Buch, in: Internationale Literatur, Moskau, 3. Jg., Nr. 4, 1933, 73-74.

Viertel, B., Das graue Tuch. Gedichte. Studienausgabe Bd. 3, hg. von Konstantin Kaiser, Wien, 1994.

Zweig, S., Die Schachnovelle, Frankfurt a. M., 2003.

\section{Sekundärliteratur}

Anders, G., Der Emigrant, in: Merkur, 16. Jg., 7, 1962, 601-622. 
Bosmajian, H., Burning books, Jefferson, NC et al., 2006.

Denkler, H. (Hg.), Colloquium zur Literaturpolitik im Dritten Reich. Das war ein Vorspiel nur..., Berlin, 1985 (=Schriftenreihe der Akademie der Künste 16).

Durzak, M. (Hg.), Die deutsche Exilliteratur 1933-1945, Stuttgart, 1973.

Friedrich, T. (Hg.), Das Vorspiel. Die Bücherverbrennungen am 10. Mai 1933. Verlauf, Folgen, Nachwirkungen. Eine Dokumentation, Berlin, 1983 (=Edition LitPol 2).

Haarmann, H. (Hg.), Das war ein Vorspiel nur... Bücherverbrennungen Deutschland 1933. Voraussetzungen und Folgen. Ausstellung der Akademie der Künste vom 8. Mai bis 3. Juli 1983, Berlin et al., 1983 (=Akademie-Katalog 137).

Knuth, R., Burning books and leveling libraries. Extreme violence and cultural destruction, Westport, Conn. et al., 2006.

Lischeid, T., Symbolische Politik. Das Ereignis der NS-Bücherverbrennung 1933 im Kontext seiner Diskursgeschichte, Heidelberg, 2001.

Mayer, H., Die deutsche Literatur am Scheiterhaufen. Bücherverbrennungen, nach 15 Jahren, in: 10. Mai 1933. Bücherverbrennungen in Deutschland und die Folgen, hg. von Walberer, Frankfurt a. M., 1983.

Rafetseder, H., Bücherverbrennungen. Die öffentliche Hinrichtung von Schriften im historischen Wandel, Wien et al., Böhlau, 1988.

Riegel, P. und W. van Rinsum, Deutsche Literaturgeschichte. Band 10. Drittes Reich und Exil, München, 2000.

Sauder, G. (Hg.), Die Bücherverbrennungen. Zum 10. Mai 1933, Wien, 1983.

Schöne, A., Göttinger Bücherverbrennung 1933. Rede am 10. Mai 1983 zur Erinnerung an die „Aktion wider den undeutschen Geist“, Göttingen, 1983.

Schoppmann, C. (Hg.), Im Fluchtgepäck die Sprache. Deutschsprachige Schriftstellerinnen im Exil, Frankfurt a. M., 1995.

Schwalm, J., „Erst Bücher, dann Menschen“. Zur Geschichte der Bücherverbrennungen, Bad Schwartau, 2006.

Schwarz, E., Exilliteratur, in: Deutsche Literatur. Eine Sozialgeschichte. Band 9. Weimarer Republik - Drittes Reich: Avantgardismus, Parteilichkeit, Exil 1918 - 1945, hg. von Glaser, Reinbek bei Hamburg, 1983, 302-317. 
Serke, J., Die verbrannten Dichter. Berichte, Texte, Bilder einer Zeit, Frankfurt am Main, 1983.

Steinbach, D. (Hg.), Literarisches Leben im Exil 1933-1945, Stuttgart 1984.

Strelka, J. P., Des Odysseus Nachfahren. Österreichische Exilliteratur seit 1938, Tübingen et al., 1999.

Treß, W., „Wider den undetuschen Geist“. Bücherverbrennungen 1933, Berlin, 2003.

Verweyen, T., Bücherverbrennungen. Eine Vorlesung aus Anlaß des 65. Jahrestages der „Aktion wider den undeutschen Geist“, Heidelberg, 2000 (=Beihefte zum Euphorion 37).

Weidemann, V., Das Buch der verbrannten Bücher, Köln, 2008.

Wegner, M., Exil und Literatur. Deutsche Schriftsteller im Ausland 19331945, Frankfurt a. M. et al., 1967. 


\section{KNJIGA IN NJEN POMEN V NEMŠKO GOVOREČEM PREGNANSTVU 1933-1945}

Ključne besede: nemško govoreče pregnanstvo, literatura pregnanstva, sežiganje knjig

\section{Povzetek}

Članek skuša s pomočjo različnih literarnih odlomkov pokazati na pomembno mesto, ki ga je imela knjiga - kot objekt in subjekt - v literarni produkciji nemško govorečih pregnancev. Kot razloge za to obravnava funkcije literature kot so antifašistični boj, izobraževanje, dokumentiranje, terapija in samoterapija. Osredotoči se na sežiganja knjig v Nemčiji leta 1933 in pokaže, kako so ti zgodovinski dogodki iz knjige naredili simbol antifašističnega pregnanstva. 


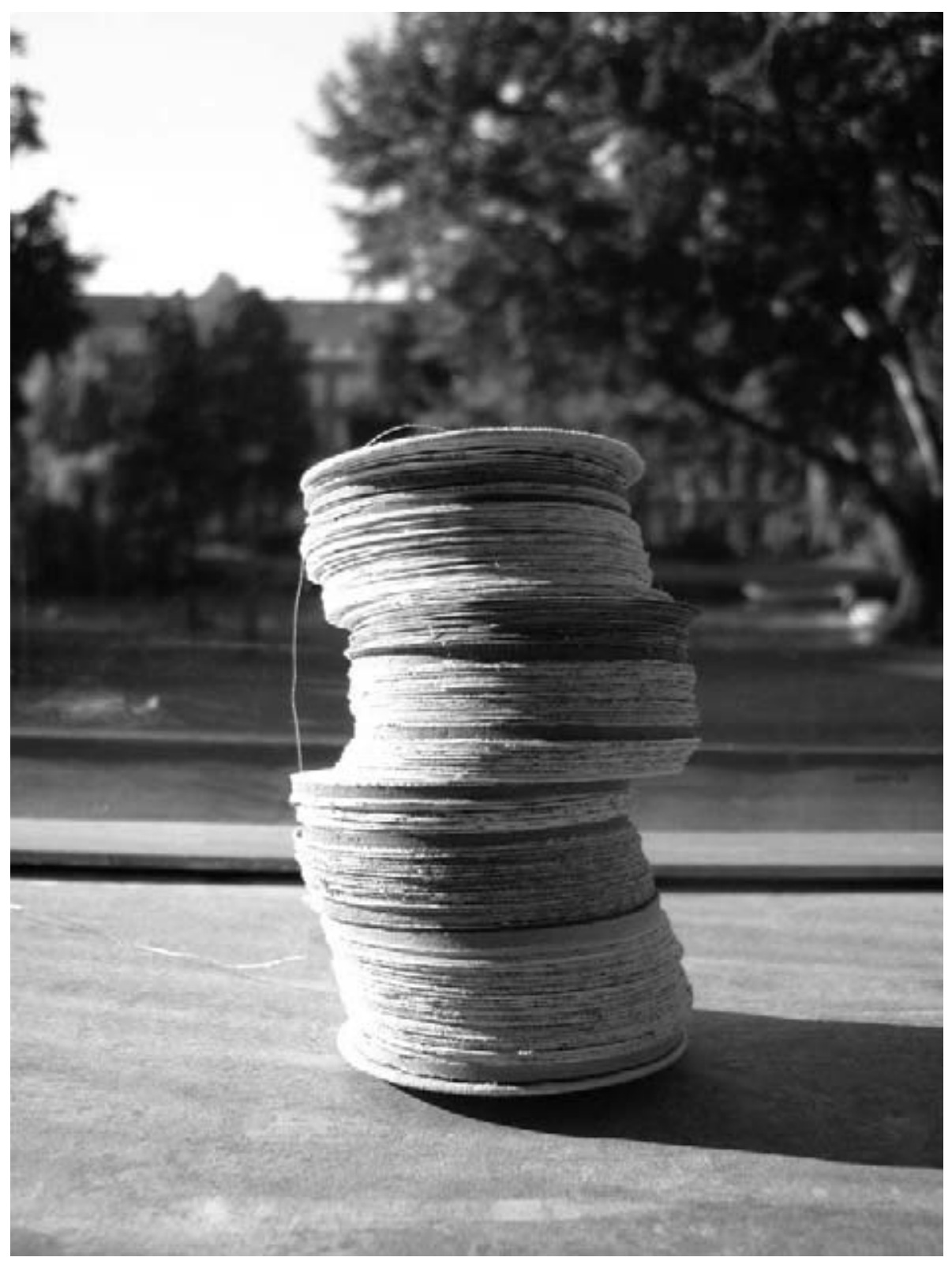

Fleur Thio: An eclectic book (2009)

"The next item in the collection is a book that answers our desire to obtain knowledge of as many different things as possible. This book consists of a chain of circular fragments of several books on different topics. I called it the 'eclectic book." 